\title{
The Gordian Knot of Glutaraldehyde-Treated Autologous Pericardium in Open Cardiac Surgery
}

\author{
Giorgio Noera \\ Cardiothoracic Surgery Unit, GVM Care \& Research Maria Cecilia Hospital, Cotignola (Ra), Italy \\ Email address: \\ giorgio.noera@gmail.com \\ To cite this article: \\ Giorgio Noera. The Gordian Knot of Glutaraldehyde-Treated Autologous Pericardium in Open Cardiac Surgery. International Journal of \\ Cardiovascular and Thoracic Surgery. Vol. 7, No. 2, 2021, pp. 13-15. doi: 10.11648/j.ijcts.20210702.11
}

Received: April 11, 2021; Accepted: May 18, 2021; Published: May 26, 2021

\begin{abstract}
Glutaraldehyde is classified as a biocide-pesticide under the hazard class for toxic substance and control laws for the human health and environmental worldwide. The regulatory bodies restrict the intended use to disinfectant or laboratory application in hospital. The parenteral pathway in human is not considered and then not allowed, for the high-risk profile, so the constraints are an integral part of the Classification Labeling and Packaging for exposure of occupational health. Contextually, there are no preclinical-safety data as Risk Related Reference (DMEL probability of occurrence and severity of adverse effect (s)/event (s) in a given population under defined exposure conditions), or Chemical Safety Reports that justify any authorization for patient consumer uses. In the context of cardiac surgery, the biomechanical stability in treating the autologous pericardium with glutaraldehyde in solution, results as a hand-built biotech for a patient valve device. Theoretically, the surgeon is a manufacturer that uses a toxic chemical compound for the own product that does not comply to the law and regulations. Contrary to pharmaceutical definition for marketing authorized drugs, the off-label not allowed use of toxic chemical derived manufacture, expose the physicians to legal vulnerability of which it is inclusive the malpractice for thepatient's informed consent.
\end{abstract}

Keywords: Glutaraldehyde, Risk Related Reference, Open Cardiac Surgery

\section{Introduction}

Glutaraldehyde (GA), possesses unique characteristics that render it one of the most effective protein crosslinking agent. GA is exclusively classified as a biocide-pesticide whose profile risk is under the hazard class for toxic substance and control laws for human health and environmental worldwide. The marketing authorization inclusive of restricted intended use, are regulated by standardized harmonized Hazard Class and Category and transcription by the manufacture under Classification Labeling and Packaging (CLP) regulation and obligation [1]. The regulatory statement for safety support is almost exclusively for occupational health linked to exposure route of skin, eyes and respiratory- digestive tract and the adverse events. The commercial GA labeling solution does not declare safety and approval form of its parenteral pathway uses [2]. However, the autologous pericardium treated with GA soaking technique in cardiovascular surgery, is supported only by wide accepted technique and not from the regulatory aspects. This work aims to frame the "prescribing" context of GA for its use, in the light of chemical toxic substance regulations and physicians legal vulnerability.

\section{Biotechnology Derived Product}

\subsection{Biotechnology}

The American Chemical Society defines biotechnology as the application of biological organisms, systems, or processes by various industries to learning about the science of life and the improvement of the value of materials and organisms such as pharmaceuticals. For the European Federation of Biotechnology, biotechnology is the integration of natural science and organisms, cells, parts thereof, and molecular analogues for products and services. Biotechnology is based on the basic biological sciences, biochemistry, cell biology, that also includes tissue technologies and conversely provides methods to support and perform basic research in biology. Using an easy and comprehensible approach, the 
wide range concept of biotechnology is a technology by which manipulated living organisms are utilized to generate useful products. Therefore, the use of the autologous pericardium should be considered as a biotechnology and not a medical device [3].

\subsection{The Derived-Products}

Biotechnology-derived drug were initially developed in the early 1980s. The first marketing authorizations were granted later in the decade. Several guidelines and points-to-consider documents have been issued by various regulatory agencies regarding safety assessment of these products. Regulatory standards for biotechnology-derived pharmaceuticals including autologous human tissues' product have generally been comparable among the European Union, Japan and United States. All regions have adopted a flexible, case-bycase, science-based approach to preclinical safety evaluation to support clinical development and marketing authorization. The primary goals of International Conference on Harmonization (ICH guideline S6 (R1) are mainly focused on toxicity, reversibility and safety parameters for monitoring $[4,5]$. Adherence to the principles is intended to improve thought consistency of the preclinical safety data. The distinction or definition between drug, toxic chemicals and delivery system is not clear. Therefore, treating the autologous pericardium with chemical substance should be considered as a procedure approach for a biotechnology derived product.

\subsubsection{Glutaraldehyde Cross Linker Agent and More}

Glutaraldehyde can cross-link proteins to create stable secondary amine linkages. However, as reported by Greg $\mathrm{T}$. Hermanson, in2013 "the homobifunctional cross linker containing an aldehyde residue at both ends of a 5-carbon chain. Its primary reactivity is toward amine groups, but the reaction may occur by more than one mechanism. Glutaraldehyde exists in a number of different forms in aqueous solution, such as hemiacetal and aldol ring structures as well as large $\alpha$-, $\beta$-unsaturated polymers containing carbon-carbon double bonds. The double bonds of these polymers can undergo an addition reaction with amines that results in covalent bond formation even without a reluctant being present. Thus, reactions with this crosslinking agent can result in indistinct conjugation products and may be difficult or impossible to reproduce or scale up as the conjugation need arises. The high reactivity and indistinct forms of glutaraldehyde make it difficult to control the size and composition of the final conjugate. Proteins cross-linked with this reagent often form substantial amounts of precipitated products due to polymerization". Moreover, the same authors report that "the glutaraldehyde in aqueous solutions can form polymers containing points of unsaturation due to aldol formation. Such $\alpha, \beta$-unsaturated glutaraldehyde polymers are highly reactive toward nucleophiles, especially primary amines. Reaction with a protein results in alkylation of available amines, forming stable secondary amine linkages. These glutaraldehyde- modified proteins still may react with other amine-containing molecules either through the Schiff base pathway or through addition at other points of unsaturation" $[6,7]$. In this context, the treated autologous pericardium with chemical substance should be considered as GA derived product.

\subsubsection{Risk and Toxicological Profile of Glutaraldehyde}

Glutaraldehyde (GA) is currently addressed as a cytotoxic agent and biocide under Regulatory Frameworks for Chemical. The profile risk, use-labeling identification and marketing authorization are exhaustively regulated within standardized harmonized Hazard Class and Category Classification CLP labeling that is almost exclusively for occupational health monitoring and establishment of safe practices for its use. In the same context, the GA toxicology profile is linked to exposure route of human skin, eyes and respiratory- digestive tracts can be inferred up to a safety tolerated Maximum Residue Limits (MRLs) of $0.001 \mathrm{ppm}$ or Minimal Risk Level of $0.00003 \mathrm{mg} / \mathrm{Kg} /$ day that it is intended for external medication only or laboratory application $(8,9)$. Recently, the OSH (Occupational Safety and Health) legislation for the protection from chemical agents provided for the identification of the indicative limit values that must be adopted by the Member States in the legislation in force (10). The fundamental concepts introduce the link to the regulations right of consumer, general population and patients. The Chemical Safety Reports statement of the various dangerous compounds and derivatives, should report the obligation of safety evaluation as the DNEL (Derived NoEffect Level, similar to the No Observed Effect Level NOAEL - for pharmaceutical products) and the DMEL-like approach (probability of occurrence and severity of adverse effect (s)/event (s) in a given population under defined exposure conditions). Exposures of biotechnology derivedtoxic-substance includes the concept of parenteral route that may have direct or submerged roots in direct contact with blood or human circulatory system. Actually, the commercial GA's CLP and marketing authorization is off guard of Chemical Safety Reports, DNEL and DML-like approach for parenteral use $[11,12]$.

\section{Glutaraldehyde in Cardiac Surgery}

\subsection{GA use for Autologous Pericardial Patch}

In cardiac surgery, for example the technique inclusive of Ozaky procedure, the aortic valve repair using neocuspidalization, require intraoperative taken fresh autologous pericardium patch before implant. Usually, the pericardium is immersed in aqueous solution with GA concentration up to $600 \mathrm{ppm}$, and then it is washed [13, 14]. After that, the treated pericardium is anchored to the patient's aortic tissues as a function of the new apparatus valvular. The operation has a time in minutes with open thorax and the manufacture does not include a toxicological test or detections for residues or bioconjugates to characterize the intraoperative safety standard of the final product. 


\subsection{GA Derived Product during Surgery}

The improvement in handling and biomechanical stability of pericardium is achieved by treating it with cross linker agent. Therefore, fixation leads to autologous tissue transformation as a part of a hybrid living apparatus. In this way, the surgical technique as conceived identifies in the surgeon a manufacturing of a heart valve using chemical compounds for its product.

\section{Conclusion}

The existing set of regulatory rules represents a gray area for GA use in open cardiac surgery. A wide accepted safe and effective of a surgical technique does not mean that the GA derived product has no other side effect or outweigh the potential risk. The interpretation of statements of regulatory support and according to the above, critical practicalities emerge of which the overlap of the definition off-label within two lines of legislations. There is a huge difference between pharmaceutical substance with preclinical and clinical trial safety studies and toxic chemical substance without Safety Report for using them [15]. Therefore, it is possible to emphasize that the manufacturer label of the glutaraldehyde in solution, is devoid of Chemical Safety Reports statement for consumer parenteral pathways. It follows that the product derived from a toxic chemical substance does not have, according to the regulations and legal aspects, the approved trial phases for human safety and authorization in the clinical practice. Therefore, the surgeon results as manufacturer of a biotechnology for the health market without any registered human safety-given-data by the supplier. At this point, the consumer rights may be violated about cytotoxic chemicals. So, the surgical technique falls under GA off label use and falls back to physician legal responsibility and the patient's informed consent. Until today, the lack of Chemical Safety Report statement, still constitute a restriction for the clinical trial approval for using this type of GA-derived product.

\section{Acknowledgements}

Accordingly, this analysis should not be used to inform any real-world decisions about the treatment or regulation of any actual substance under either regulatory scheme, as that would require a more detailed analysis. The author declares that he has no conflicts of interest.

\section{References}

[1] NHI: Glutaraldehyde [Internet]. Bethesda: National Library of Medicine. PubChem (c) Available from: https://pubchem.ncbi.nlm.nih.gov/compound/glutaraldehyde.

[2] European Chemical Agency (2020) Consolidated version of the CLP Regulation Commission Regulation (EU) No 2019/521: Available from https://echa.europa.eu/it/rregulations/clp/legislation.
[3] Cavagnaro A. Preclinical safety evaluation of biotechnologyderived pharmaceuticals (2002) Nature Reviews Drug Discovery (1), 469-475.

[4] European Commission. Medical Device Coordination Group Document (2020) MDCH 2020-12 Guidance. Available from: https://ec.europa.eu/health/sites/health/files/md_sector/docs/m d_mdcg_2020-12_guidance_transitional_provisions_en.pdf.

[5] EMA: ICH S6 (R1) Preclinical safety evaluation of biotechnology- derived pharmaceuticals. 2011. European Medical Agency. Available from: https://www.ema.europa.eu/en/documents/scientificguideline/ich-s6r1-preclinical-safety-evaluationbiotechnology-derived-pharmaceuticals-step-5_en.pdf.

[6] Hermanson G. T. (2013) Bioconjugates Technique Third Edition Elsevier ISBN 978-0-12-382239-0.

[7] Migneault I., Dartiguenave, C., Bertrand, M. J., Waldro, C. (2004) Glutaraldehyde: behavior in aqueous solution, reaction with proteins, and application to enzyme crosslinking. Bio, Techniques 37: 790-802.

[8] ATSDR: Glutaraldehyde. Atlanta: Agency for Toxic Substances and Disease Registry Division of Toxicology and Human Health Sciences Environmental Toxicology Branch. (C)2017. Available from: https://www.atsdr.cdc.gov/Tox Profiles/tp208-p.pd4.

[9] ATSDR: Glutaraldehyde A-1 Appendix A Minimal Risk Level and Worksheet. The Comprehensive Environmental Response, Compensation, and Liability Act (CERCLA) [42 U.S.C] [Internet]. Atlanta:Agency for Toxic Substances and Disease Registry; Available from: https://www.atsdr.cdc.gov/toxprofiles/tp208.pdf.

[10] EU-OSHA Agency European Union Directive 98/24/EC risks related to chemical agents at work Available from: https://osha. europa.eu/en/legislation/directives/75.

[11] Yanoa, K., Watanabea, N., Tsuyukic, K. Ikawac, T., Kasanukib, H., Yamato, M. (2015) Regulatory approval for autologous human cells and tissue products in the United States, the European Union, and Japan. Regenerative Therapy 1, 45-56.

[12] European Medical Agency. (2019) First guidance on new rules for certain medical devices Available from https://www.ema.europa.eu/en/news/first-guidance-new-rulescertain-medical-devices.

[13] Pirola S., Mastroiacovo G., Arlati FG., Mastardini G., Bonomi A., : Penza E., Polvani G.(2020) Single Center Five Years' Experience of Ozaki Procedure: Midterm Follow-up. Available https://doi.org/10.1016/j.athoracsur.2020.08.039.

[14] Sá M. P. B. O., Perazzo Á. M., Zhigalov K., Komarov R., Kadyraliev B., Enginoev S., Ennker J., Popov A. F., Quarto C., Weymann A., Lima R. C. (2019) Aortic Valve Neocuspidalization with Glutaraldehyde-Treated Autologous Pericardium (Ozaki Procedure) - A Promising Surgical Technique Braz J Cardiovasc Surg. 1; 34 (5): 610-614.

[15] Wittich C. M., Burkle C. M., W. L. Lanier. (2012) Ten Common Questions (and Their Answers) About Off-label Drug Use Mayo Clin Proc.; 87 (10): 982-990. 